\title{
Research on Internal combustion engine speed processing method and its parameters optimization
}

\author{
Qiu-yuan Wang ${ }^{1, a}$, Fu-jun Zhang ${ }^{1, b}$, Zhe Zuo ${ }^{1, c}$ \\ ${ }^{1}$ Beijing Institute of Technology, Beijing, 10081, P.R. China \\ a'wangqiuyuan2009@sina.com, bfj123@bit.edu.cn, 'czuzeus@gmail.com
}

\begin{abstract}
Keywords: Engine speed processing, Matlab/xPC HILS platform, Simulink model, Digital filtering. Abstract: The accurate and available engine rotate speed signal is quite essential to engine control, fault diagnosis, etc. This paper analyses the parameters optimization towards common processing methods of engine rotate speed, aiming at internal combustion engine closed-loop control. Basing on the Matlab/xPC target simulation platform with closed-loop controlling self-designed engine speed simulation device, it puts forward a series of simulink model of the processing methods and achieves a way to process real rotae speed signals. Finally it realised a steady condition of closed-loop control after relevant optimization, which have tested the feasibility of parameter optimization of rotate speed processing by comparing the practical results of different speed processing methods with different parameters. The experiment result indicates that it is instructive in the parameter setting for internal combustion engine speed processing method.
\end{abstract}

\section{Introduction}

The engine rotate speed signal is an information-rich source of the operational state of internal combustion engines, the study of which is very important for realising fault diagnosis, closed-loop feedback control and engine control. It is a result of the joint effect of combustion gas, reciprocating inertia and loads ${ }^{[1]}$, etc. Aiming at different application purposes of engine speed, we can get accurate and available engine rotate speed signals that is essential to the following work such as speed calculation and engine control. Therefore, the processing towards engine speed signal is too valuable to be neglected.

Depending on the application purpose and the measurement principle, rotate speed can be divided into three categories: instantaneous speed, cycle speed and average speed. Instantaneous speed is the average value angular velocity for covering a tiny crank angle, which seems to mostly nearly to the velocity of every single trice of rotating. It is utilized to reflect the uniformity and stability of the each cylinder of the engine, and to demonstrate the functional parameters such as torque, combustion pressure of each chamber, and the indicated thermal efficiency from the engine. Cycle speed stands for the average of angular velocity in a single engine cycle, which can be employeed to describe two corresponding points on successive cycles, and is of great importance in the study of stability and transient working conditions of internal combustion engines. Average speed, in other words, the average value angular velocity of rotating in a period of time (regularly more than one cycle) is often used to describe the speed of an engine under steady working conditions, and also can be an reference variable of internal combustion engine control such as the speed closed-loop feedback control.

Basing on the general characteristic of the rotate speed of internal combustion engines and aiming at the common engine speed processing methods of the speed closed-loop control, this paper analyses the parameters optimization of the methods. Basing on the Matlab/xPC target simulation platform with closed-loop controlling self-designed engine speed simulation device, this paper puts forward a series of simulink model of the processing methods and achieves a way to process real rotate speed signals, with magnetoelectric sensor untouched measuring toothed gear's speed. It also tested the feasibility of parameter optimization of rotate speed processing, compared the practical results of different speed processing methods, and finally realised a steady condition of closed-loop control after relevant optimization. 


\section{Important Influential Factors of Engine Speed Processing Methods}

The accurate measurement of rotate speed is the premise of engine speed processing, the main measuring methods of which is high frequency counting method. The method uses counters to acquire pulse signals that have been conditioned and get the cycle time by calculating the sample size between every two pulse repetition. The signal period acquired from the method corresponds to one tooth of toothed gear, and we can calculate the rotate speed by counting out the number of tooth that a toothed gear has. The accuracy of the signal period depends on the sample frequency. As the rotate speed rises, the sample frequency shall be increased correspondently. If the frequency of counter is not high enough for sampling requirements, it may cause large error of the signal period and even lead to miss the sampling tooth. Therefore, it shall choose sampling frequency as high as possible.

Another factor that matters is the ratio of synchronization. When the Instantaneous speed of internal combustion engine is needed, a high standard of measuring and calculating synchronization of rotate speed must be achieved. Thus, high order integral and time delay link, as well as the big delay after processing must be avoided to guarantee the effective and desirable processing. For studies of engine control or running conditions, it shows a relatively lower requirement of cycle speed and average speed, but a distorted rotate speed during a correponding time is also intolerant, if we want to control the error within an accepted range of scale.

\section{The Common Processing Method of Engine Speed and Parameter Optimization}

The combustion-gas pressure and the mechanical rotating non-uniform create vibration and obstruction, which might badly influence the speed signals. Meanwhile, the measurement of signals through the data acquisition system is not absolutely faultless. Under the influence of the electronic system and the internal combustion engine itself, a large amount of noise is predictable. Thus a proper treatment of rotate speed is indispensable in every sense. This paper will give an analysis to the common treatment towards rotate speed and relevant parameter optimization. Generally, the common methods involve outlier eliminating, digital filtering and mean value method. Interpolation method may be also employeed under some circumstances that require a higher accuracy of Instantaneous speed.

Outlier eliminating method, as is implied by the name, is to deliberately eliminate the conspicuously unconventional data from the raw rotational speed signals. Amplitude and frequency of change that shows a pattern of wider fluctuation is due to vibration or other interference signals, the impacts of which are difficult to be thoroughly eliminated by traditional filtering method. Since Instantaneous speed has continuity and relative stability, hence the difference of adjacent rotative speed in a high sampling rate situation shall maintain a minimum jump amongst data. Utilizing this feature, we apply error discriminate criterion to distinguish outlier. Statistically, Paŭta Criterion, Chauvenet Criterion, Grubbs Criterion and Dixon Criterion are the main methodologies to achieve the rejecting: the final three are mainly performed by consulting the distribution table, which makes the Paúta criterion the most straightforward and commonly used method to identify outlier under normal distribution. The basic idea of Paŭta criterion is to set the triple value of the standard error of measurement as the benchmark, when the degree of confidence of $97 \%$ is given. The equation is shown as Eq. 1.

$$
\left|x_{i}-\bar{x}\right|>3 \sigma \text {. }
$$

If Eq. 1 comes into existence, we can conclude that $x_{i}$ is a outlier and must be eliminated. In other words, the probability that the absolute value of the residual of $x_{i}$ is greater than $3 \sigma$ is approximately $0.27 \%$. In a limited sample size, the situation stated above is almost an impossible event. In the Eq. 1 , the estimate value of standard deviation $\sigma, S$, is calculated by the Bezier Equation, as can be seen form Eq. 2. 


$$
S=\sqrt{\frac{\sum_{i=1}^{n}\left(X_{i}-\bar{X}\right)^{2}}{n-1}} .
$$

$n$ is a limited number of samples, which is an important influential parameter for the result of outlier eliminating. When the number of samples less than 10, all the absolute values of the residual are less than $3 S$ and Paǔta Criterion fails to distinguish, which shows Paŭta Criterion only apply to the situation that the number of samples greater than $10^{[2,3]}$. In order to eliminate all the outliers within sampled data which actually contain more than one outlier, the sample size should be as big as possible. For the sake of sample numbers, we discuss further and put forward the conception of the error rate of outlier eliminating. For independent identically distributed samples, the probability of a single normal sample locates outside the range of $\pm 3 \sigma$ is about $0.27 \%$. With the increasing number of samples, the error rate of normal samples being eliminated also rising. Only when all the normal samples fall in the range of $\pm 3 \sigma$, the result that no one normal sample has been eliminated is what we do expect. The error rate equation of Paǔta Criterion is shown as Eq. 3.

$$
P_{e}=1-(1-\mathrm{P})^{n}=1-(1-0.0027)^{n} \text {. }
$$

From the Eq. 3, we know the fact that sample size is bigger and then the error rate is higher. Generally, we limit the error rate within $4 \%$ to ensure the reliability and authenticity after data processing and finally choose $n=15$. Considering about the large sample size takes up too much storage space and may bring about serious data delay, the above optimization figure is also reasonable. Meanwhile, when calculating, in order to ensure real-time of the output, this paper first one-time takes 15 samples from the initial samples to get average and then use a next new sample to replace the oldest one in the 15 figures successively, guaranteeing at every sample time there is a new average output and 15 sample size of data. In terms of outliers eliminating, the average of two former normal figures which have been corrected replaces the outlier to output, instead of deleting directly.

Digital filtering means that an algorithm or a program convert the input of digital signals to the required output signals according to the reservation form. With many outstanding advantages such as high stability, high precision, large flexibility, digital filtering is widely use in speed processing. This paper here focuses on discussing and analyzing moving filtering and farrow filtering, which are most applied and easy to operate belonging to digital filtering. Moving filtering means sliding one by one and taking $m$ numbers of contiguous data to weighted average, which can smooth the data. General equation for moving filtering is shown as Eq. 4.

$$
f_{k}=y_{k}=\sum_{i=-q}^{p} w_{i} y_{k+i}, k=q+1, q+2, \mathrm{~L}, N-p
$$

In the Eq. $4, f_{k}$ is the replacement value of $y_{k}$ after weighted averaging. $w_{i}$ is the weight coefficient, and $\sum_{i=-q}^{p} w_{i}=1 . p$ and $q$ are any positive integer less than $m$, and $p+q+1=m$. Different values of these parameters form different types of the moving filtering. General so-called moving filtering is actually the equal weighted central moving average method, as $p=q=n$, $w_{i}=\frac{1}{2 n+1}$. The equation is shown as Eq. 5 .

$$
f_{k}=y_{k}=\frac{1}{2 n+1} \sum_{i=-n}^{n} y_{k+i}, k=n+1, n+2, \mathrm{~L}, N-n ; \quad m=2 n+1 \text {. }
$$

According to the different parameter values, besides the above, there are asymmetric moving average method, equal weighted endpoint moving average method and so on. The smoothing result of the data also depends on the parameter values which mainly are the number $m$ of contiguous data and weight coefficient ${ }^{w_{i}}$. If $m$ is too large, the normal fluctuation of the data will be smoothed, otherwise too much interference fluctuation will be retained; while ${ }^{w_{i}}$ has the similar effect as $m$, the selection 
of $w_{i}$ shall accord to the degree of the smooth effect which the different distances from

$y_{k}$ determined. At present there is no theoretical basis for the parameter selection besides some study applying the genetic algorithm ${ }^{[4]}$ which is immaturational yet. Therefore it generally relys on experience to select parameters of moving filtering as reasonable as possible. Aiming at dynamic test data preprocessing, the equal weighted center moving average method is the most suitable method, with $m=5$ or $m=7$. If data is required more smoothly, $m=9$ or $m=11 .{ }^{[5,6]}$ Another common digital filtering - Farrow filtering is a kind of FIR filtering method which realize clock signal fractional time delay and variable delay length interpolation. Based on the linear interpolation, the Farrow filtering equation is shown as Eq. 6.

$$
y[k]=(1-a) x[k-1]+a x[k], 0 \leq a \leq 1 .
$$

With the different values of $a$ in the Eq. 6, interpolation delay can be limited between $0 \sim 1$. Combining with the above analysis of the moving filtering, farrow filtering also belongs to moving filtering, the essence of which is the endpoint smoothing filtering with $m=2$. Basing on the Eq. 6 and comprehensively considering about speed delay and smoothing effect, the parameter $a$ should be equal to 0.5 .

Mean value method means to output the average rotate speed of a period of time, which is equivalent to lowering the sample frequency, including toothed gear average and integer periods average. Toothed gear average is used chiefly in the processing of instantaneous speed to downsize the deviation caused by low manufacture accuracy of the gear rim and low A/D conversion accuracy. The average teeth amount shall avoid the submultiple of the total teeth numbers to prevent a fixed quantity combination. Integer periods average usually stands for an average time domain of integer cycle of internal combustion engines. Considering the impacts of reciprocating inertia force, torsional vibration of crankshaft, mechanical whirling damping and combustion gas bore on speed fluctuation, the cycle period is often calculated in the form of cycles. So it is necessary to average the time domain of integer periods and filter the cycle interference signals during the study of cyclic fluctuation and the rotate speed control. The mean value method within time domains actually is acting the role of a comb filter: it is a selective system for desired signals and their miltiple-frequency ones, while for the cycling signals that owns undisired frequency, it provides serious restriction effects. In consideration about that speed precision will reduce after averaging, it shall take average processing only during a single cycle under the premise of satisfying the requirement of control.

\section{The Realization of Engine Rotate Speed Processing based on Simulink and Platform Tests}

The outline of testing platform

This paper set up a Matlab/xPC Target semi-physical real time simulation platform to verify and compare the parameter optimization results of rotate speed processing, which is based on simulink. We use a host-target machine mode and NI data acquisition system to acquire and output rotate speed control signals. Basing on the above simulation platform with closed-loop controlling self-designed engine speed simulation device, this paper puts forward a series of simulink model of the processing methods and achieves a way to process real rotate speed signals, with magnetoelectric sensor untouched measuring toothed gear's speed. The frequency of the measuring counter is $80 \mathrm{MHz}$, which has exceeded the required maximum sampling frequency. The testing platform is shown in Fig. 1 and the engine speed simulation device is shown in Fig. 2.

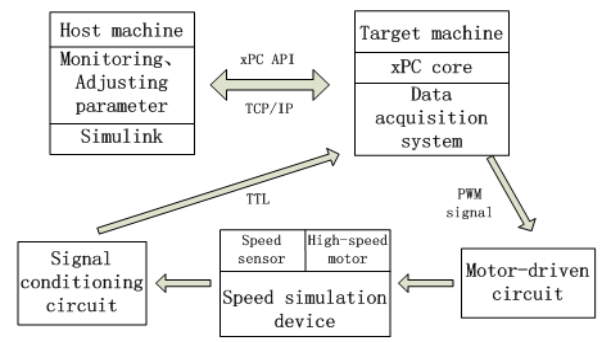

Fig. 1. The testing platform

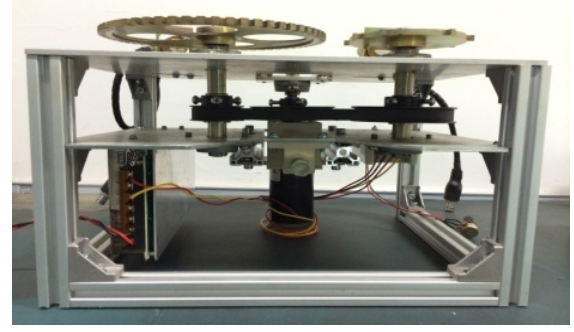

Fig. 2. The engine speed simulation device 
The realization of rotate speed processing based on simulink

The simulink model of the testing platform includes speed measuring module, speed calculating module, speed processing module and PID control module. This paper mainly aims at the speed processing module to introduce detailedly, while other modules has been solidified and don't describe here. This needs to be stressed that the values of part parameters in the sketches are just for demonstration, not the optimization of parameters.

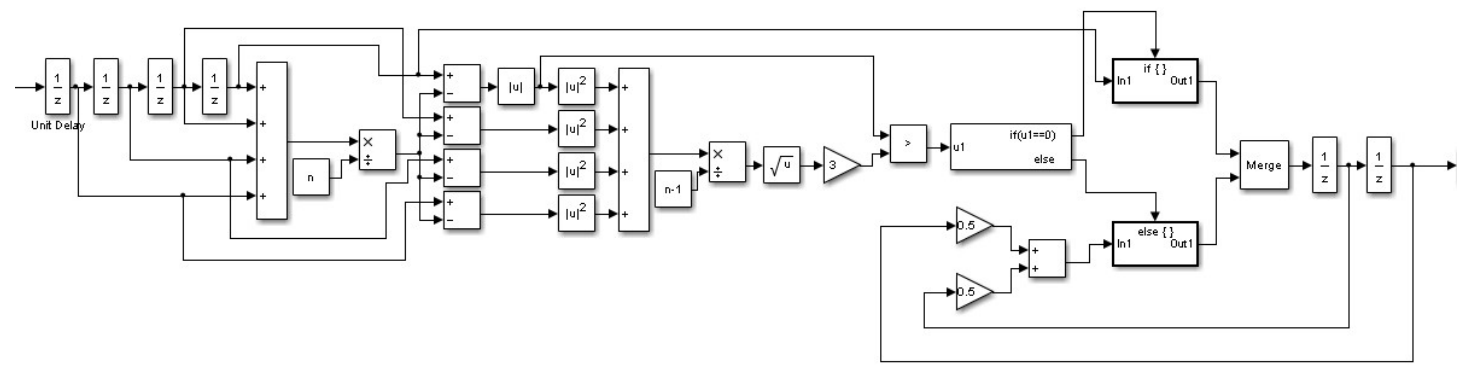

Fig. 3. The simulink module of Paŭta Criterion, $n=4$

The outlier eliminating method apply Paŭta Criterion. The simulink module of Paŭta Criterion with $n=4$ is shown in Fig. 3. If $n=15$, it just need to add Unit Delay modules. The corresponding equation is Eq. 1 and Eq. 2. Their meanings have detailed above.

$$
\begin{aligned}
& \left|x_{i}-\bar{x}\right|>3 \sigma . \\
& S=\sqrt{\frac{\sum_{i=1}^{n}\left(X_{i}-\bar{X}\right)^{2}}{n-1} .} .
\end{aligned}
$$

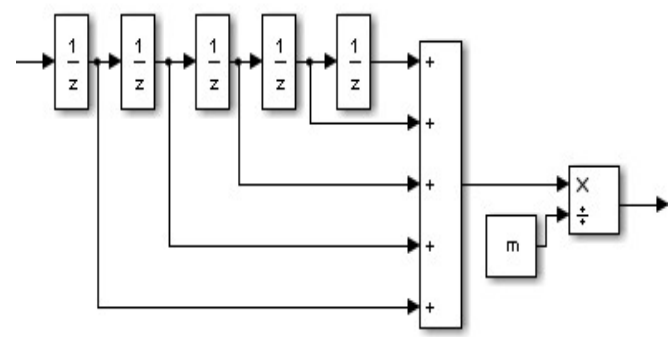

Fig. 4. The simulink module of equal weighted moving average method, $m=5$

The application of digital filtering includes moving filtering and farrow filtering. The simulink modules of these methods are shown in Fig. 4 and Fig. 5 respectively. The equation of equal weighted moving average method with $m=5$ is Eq. 5. The essence of farrow filtering is the endpoint smoothing filtering with $m=2$, the equation of which is shown as Eq. 6 .

$$
\begin{aligned}
& f_{k}=y_{k}=\frac{1}{2 n+1} \sum_{i=-n}^{n} y_{k+i}, k=n+1, n+2, \mathrm{~L}, N-n ; m=2 n+1 . \\
& y[k]=(1-a) x[k-1]+a x[k], 0 \leq a \leq 1 .
\end{aligned}
$$




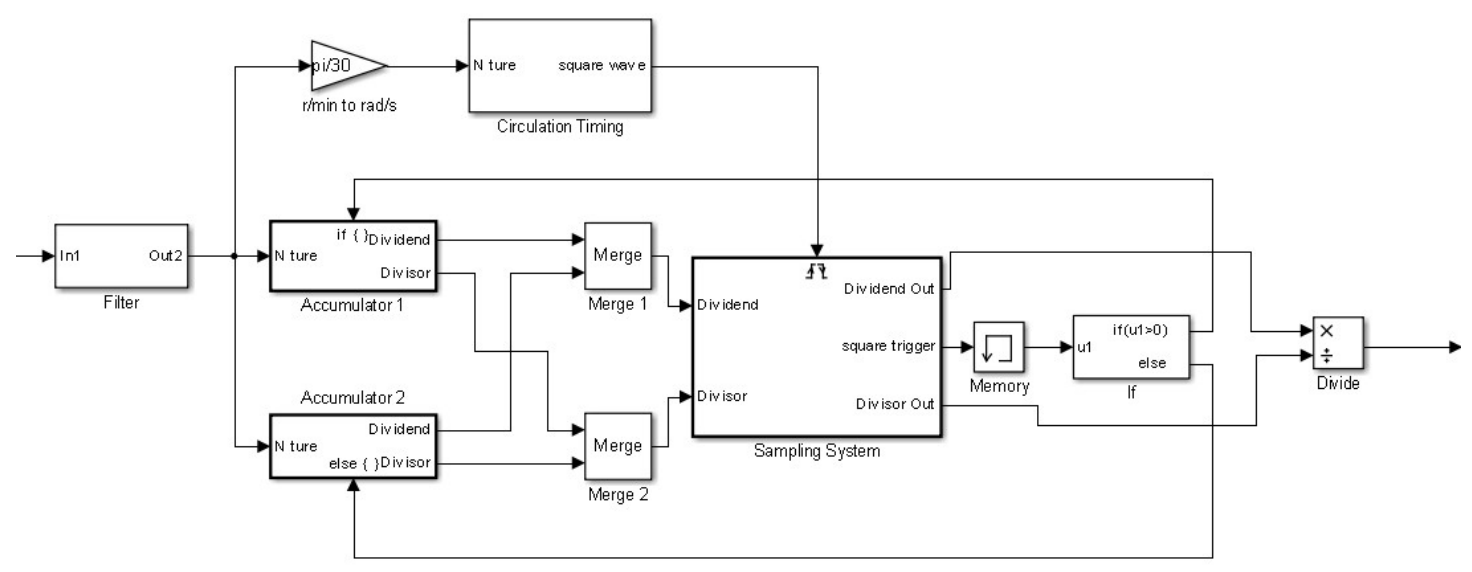

Fig. 6. The simulink module of mean value method

The whole simulink module of mean value method is shown in Fig. 6, including the subsystems such as Circulation Timing, Sampling System、Accumulator and corresponding modules about switching the Accumulators.

Circulation Timing

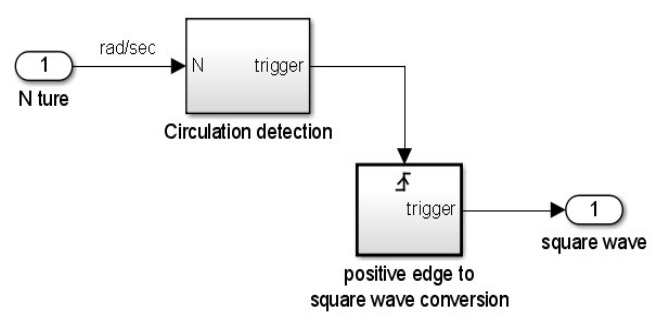

Fig. 7. Circulation Timing Subsystem
Circulation Detection

Produce one pulse per circulation.

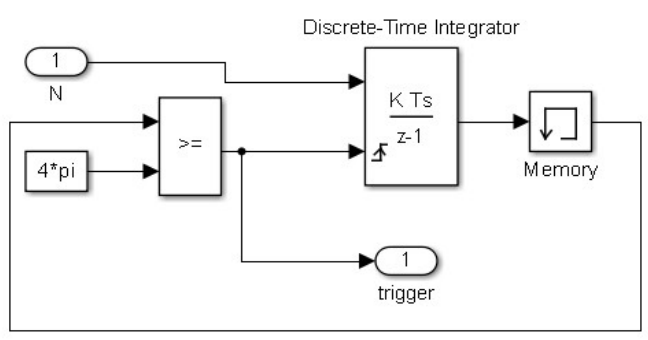

Fig. 8. Circulation Detection Subsystem

Circulation Timing Subsystem is shown in Fig. 7. Integral radian which integrate from $n \mathrm{rad} / \mathrm{sec}$ compares with fixed circulation radian. When integral radian equal to or greater than fixed circulation radian, Circulation Detection Subsystem which is shown in Fig. 8 creates a trigger signal and resets the discrete integrator. The time interval between two triggers is the time during which the integrator integrate from zero to fixed circulation radian. The Memory module plays a role in avoiding algebraic loop. After creating trigger signal, the positive edge to square wave conversion subsystem convert three triggers to one square wave. The time interval between two triggers is the high level or low level of the square wave.

Sampling System

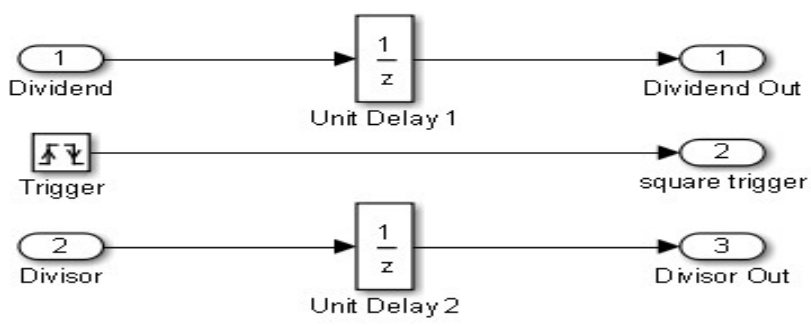

Fig. 9. The simulink module of Sampling System

Sampling System, which is shown in Fig. 9, is a sampling trigger subsystem that is triggered by rising edge and falling edge of square wave. In the system, when the trigger module is triggered, it outputs and maintains the high or low level of square wave which regards as the judging signal of switching the Accumulators. 


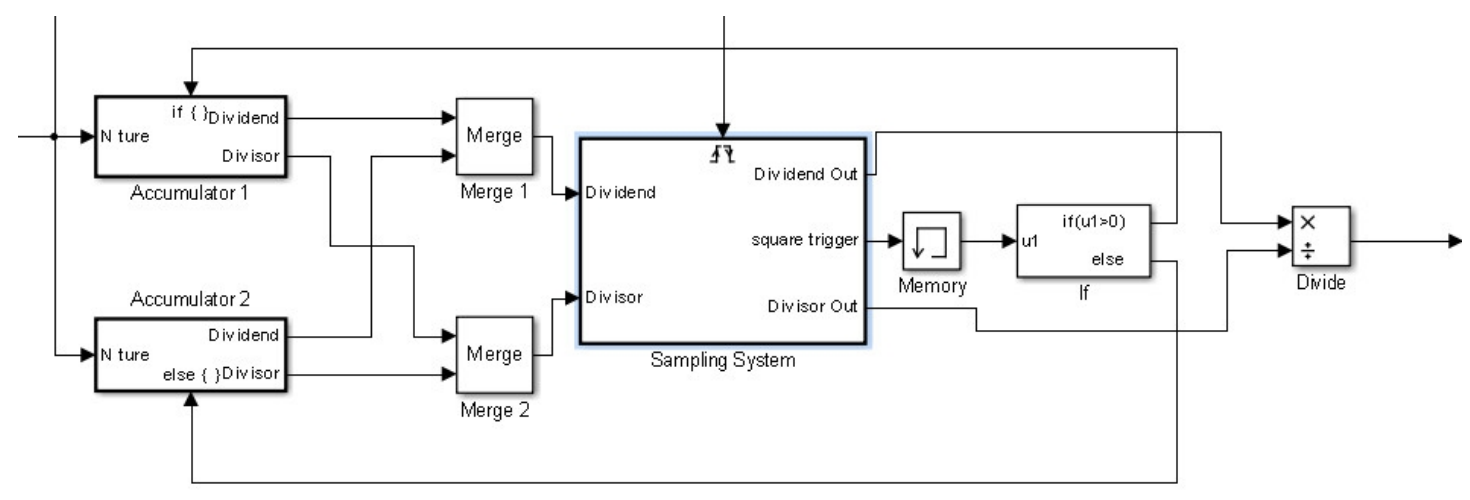

Fig. 10. Accumulator Switching System

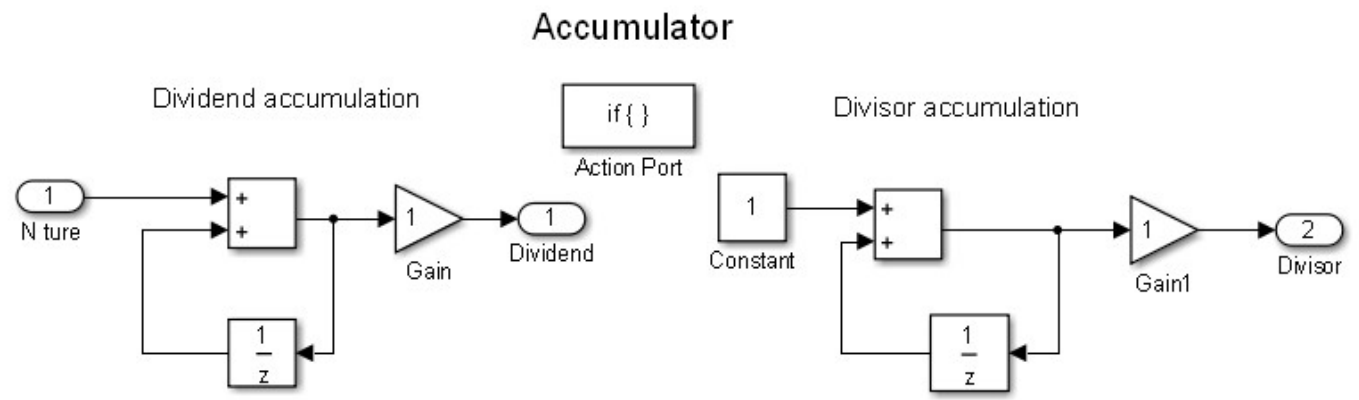

Fig. 11. The simulink module of Accumulator Subsystem

The If module calls the Accumulator 1 and Accumulator 2 respectively by judging the high and low level of square wave to realize the switch of two accumulators, which is shown in Fig. 10. The simulink module of Accumulator Subsystem is shown in Fig. 11. Accumulators accumulate the Dividend and the Divisor at the same time. The Dividend is the sampling speed and the Divisor is the number of the samples. Finally, the Divide module take average to acquire the mean speed.

Experimental verification of the parameter optimization

On the premise of the module solidification such as speed measuring module, speed calculating module and PID control module, we suppose that every parameter has reached its optimal value, besides the speed processing module. In this background, we use the Matlab/xPC Target platform to vertify the sequel of parameter optimization and to get a more stable close-loop speed controlling effect. The difference between the target speed and real acquired speed is the fluctuation of $e$ which input to PID. We assume that the controlling parameters are fixed, in the circumstance, each effect of speed processing is corresponding to a specific $e$ fluctuation, hence the differences of fluctuation output by PID and eventually reflect to the fluctuation of real speed of the simulation device. We can justify the result of speed processing by acquiring and comparing the real rotate speed. $1000 \mathrm{r} / \mathrm{min}$ is set as the target speed of the real rotation. What follows is the contrast analysis of speed signals that gained from different speed processing.

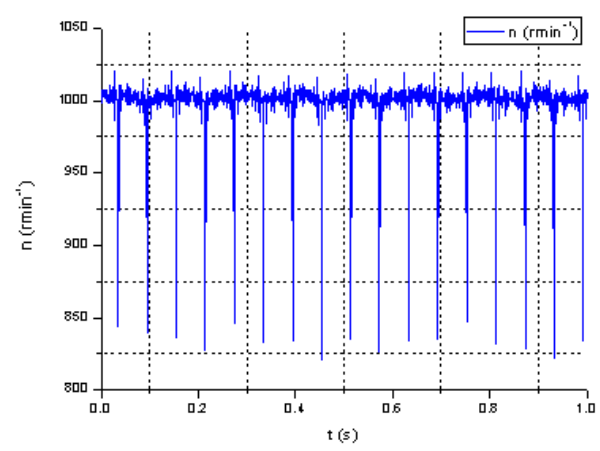

Fig. 12. Speed signal before processing

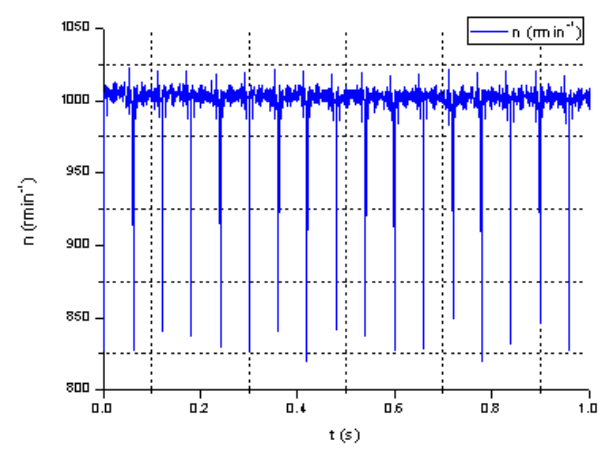

Fig. 13. Processing result of Paŭta Criterion, $n=9$ 

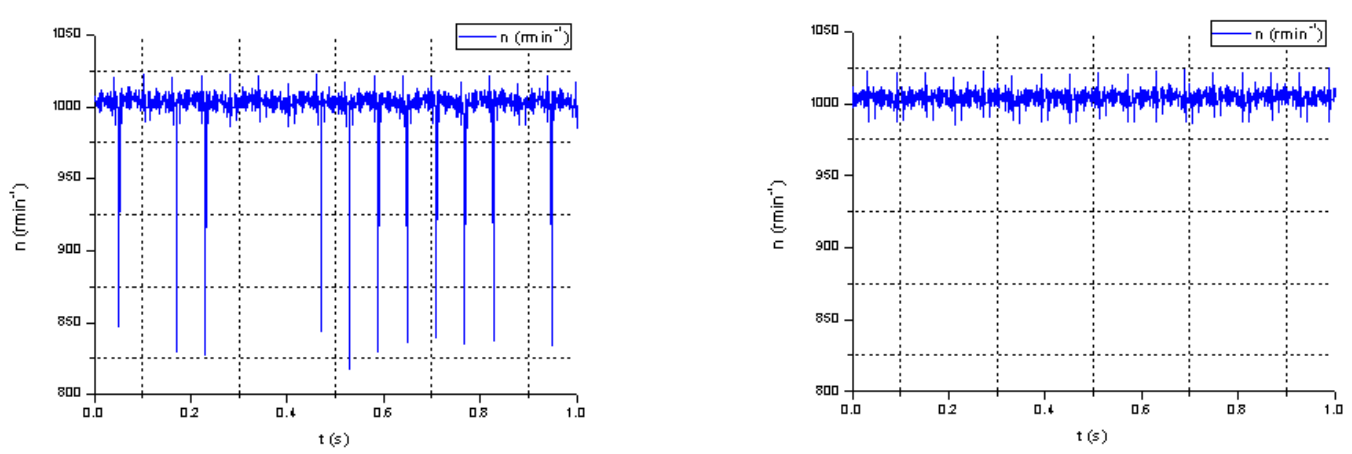

Fig. 14. Processing result of Paŭta Criterion, Fig. 15. Processing result of Paŭta Criterion, $\mathrm{n}=12$ $\mathrm{n}=15$

As shown in Fig. 12, obviously, the raw speed fluctuation signals after collected, measured and calculated maintains cyclic interference error that exceed the normal error and caused by the mechanical devices. We use the Paǔta Criterion to eliminated the outliers, and get the result shown in Fig. 13, when the sample size $n=9$, outliers remained uncleared, the Paŭta Criterion failed. When $n=12$, as shown in Fig. 14, only part of outliers are excluded, so the Paǔta Criterion is not functioning properly. So we concluded that the sample size shall be enlarged as far as possible in the reasonable range. When $n=15$, as shown in Fig. 15, the effect of the elimination is obvious and the result supported our expectation above.

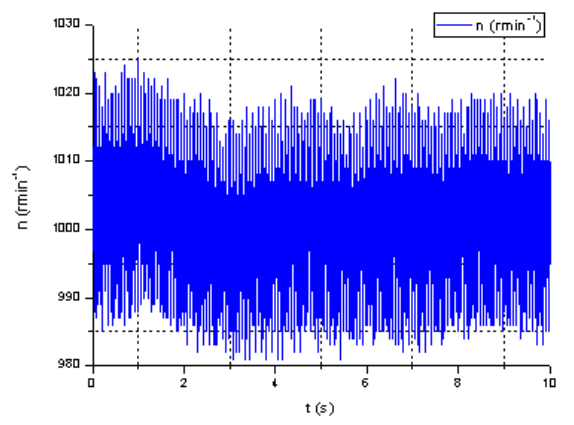

Fig. 16. Speed signal before digital filtering

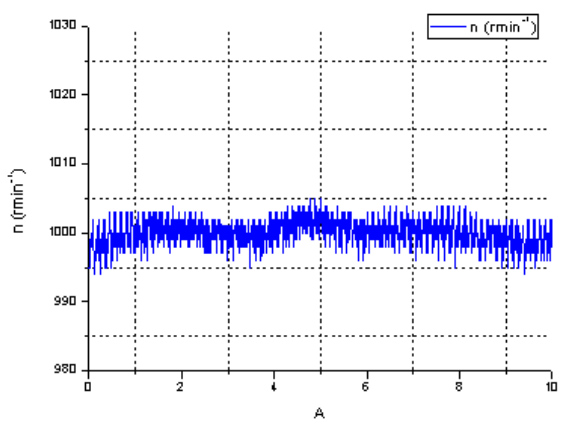

Fig. 18. Processing result of equal weighted central moving average method, $\mathrm{m}=11$

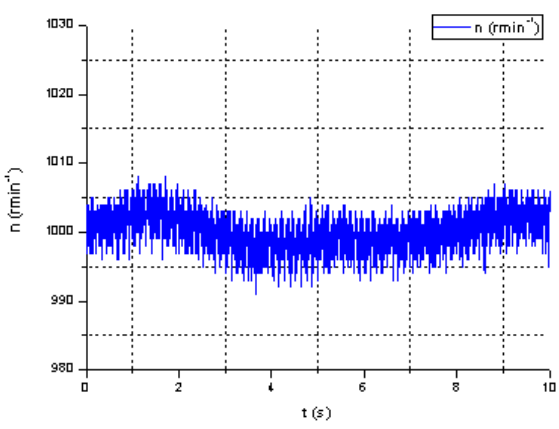

Fig. 17. Processing result of equal weighted central moving average method, $\mathrm{m}=5$

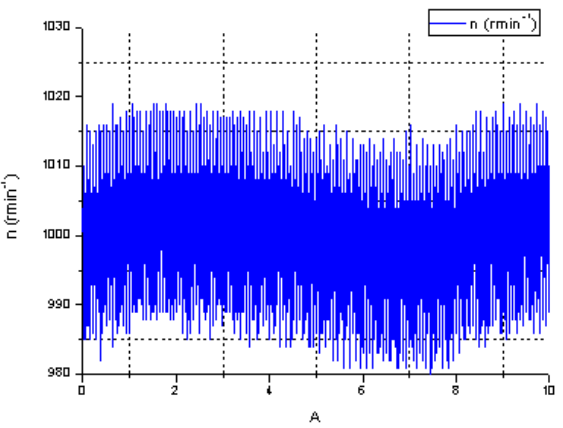

Fig. 19. Processing result of farrow filtering, $\mathrm{a}=0.1$ 


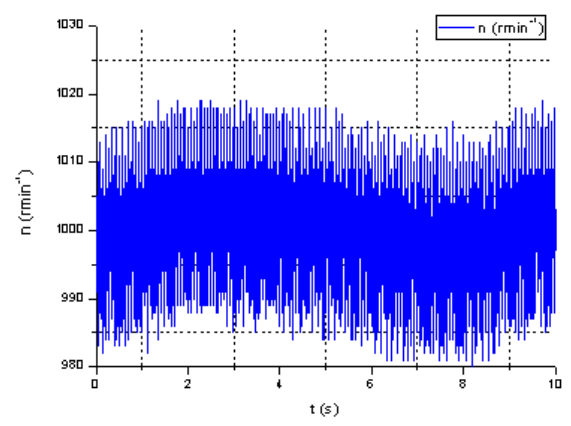

Fig. 20. Processing result of farrow filtering, Fig. 21. Processing result of farrow filtering, $\mathrm{a}=0.9$

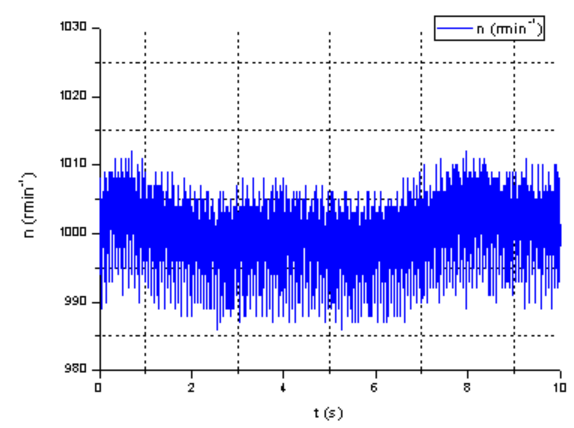
$\mathrm{a}=0.5$

Fig. 16 shows the speed signal before digital filtering and after the outliers eliminating. Based on the equal weighted central moving average method, the most common-used average smoothing filtering method, Fig. 17 and Fig. 18 show the filtering effects when $m=5$ and $m=11$ respectively, while $m$ stands for the average amount of consecutive data. Considering the real smoothing effect, data reliability, and storage space taken, the result is more appropriate when $m=11$ which is shown in Fig. 18 and meets the requirements. Farrow Filtering is another common method. When $a=0.1$, the result can be found in Fig. 19. When $a$ changes to 0.9, it is illustrated in Fig. 20. By comparing these two, the result shows only a slight difference, but, when $a=0.9$, less delay effect seems to be what matters, by analyzing Eq. 6. Fig. 21 shows the result when $a=0.5$, considering the delay it caused and real smoothing effect, we take $a$ as 0.5 eventually, which supported our expectation above.

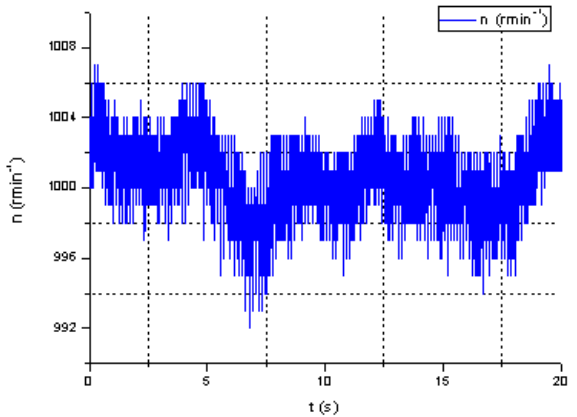

Fig. 22. Speed signal before mean value processing

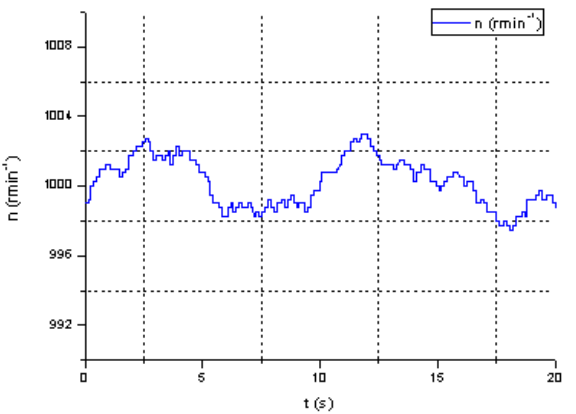

Fig. 23. Processing result of mean value method with a single cycle

After the outlier eliminating and digital filtering processing, the fluctuation of the optimized speed can be limited within $\pm 6 \mathrm{r} / \mathrm{min}$, as can be seen from Fig. 22. But the fluctuation frequency is relatively high, and the result is still mixed with periodic interference, which is not beneficial to a steady close-loop speed control. If we strike an averaging treatment upon a single cycle of rotate speed, as can be seen from Fig. 23, the fluctuation amplitude and frequency have reduced greatly compare with those in Fig. 22. Considering the practical effect of single cycle averaging method and the delay of close-loop control in multi-cycles circumstances, the processing we mentioned above can truly satisfy the required optimization in the process of close-loop control, which has supported our expectation. Finally, as a combination of outlier eliminating, digital filtering, mean value method, the solution of this paper has achieved the desired close-loop control effect while parameters are properly optimized, as shown in Fig. 23.

\section{Conclusions}

This paper analyses the parameters optimization towards common processing methods of engine rotate speed, by employing the Matlab/xPC semi-physical simulation platform and close-loop controlling engine simulation device. It also puts forward a series of simulink model of the processing methods and achieves a way to process real rotate speed signals, verifying the feasibility of parameter 
optimization and comparing the practical results of different speed processing methods. Finally it realised a steady condition of closed-loop control after relevant optimization. The experiments have presented the outlier eliminating, digital filtering and mean value method as practical in the process of optimization towards speed processing, which is instructive in the parameter setting, given the internal combustion engine closed-loop control as the application target.

\section{References}

[1] Zhe Zuo, Ying Huang, Chang-lu Zhao, Research on Integrate Robust control Method for High-power Variable-Speed Governor of Diesel Engine by Loop-Shaping theory, CCDC, Xuzhou, China (2010).

[2] Yan-yan Xiong, Xian-qiu Wu, Comparison and Application of Four Gross Error Criterions, J. Physical Experiment of College. 23 (2010) 66 -68. In Chinese.

[3] Su-jian Lai, Xiao-xiong Jin, Wei Peng. Research on Wrong points eliminating method of signal pre-processing, J. Journal of Jiamusi University. 29 (2011) 333-336. In Chinese.

[4] Ming-kai Huang. Research on Parameters of Moving average Digital filtering, J. Journal of Jimei University. 11 (2006) 381-384. In Chinese.

[5] Xuan Yang, Sheng Li, Jun-li Deng. The Improvement of Moving Average Filter in Numerical Control, J. Power Electronics. 49 (2014) 68-70.

[6] Hong-ye Lin. Data Processing of Dynamic Measurement, Beijing Institute of Technology Publishing, Beijing 1995. In Chinese. 